\title{
EXTRAÇÃO E FITODISPONIBILIDADE DE METAIS EM RESPOSTA À ADIÇÃO DE LODO DE ESGOTO NO SOLO'
}

\author{
Marcia Aparecida Simonete 2,$4 ;$ Jorge de Castro Kiehli* \\ ${ }^{2}$ Pós-Graduanda em Solos e Nutrição de Plantas - USP/ESALQ. \\ ${ }_{4}^{3}$ Depto. de Solos e Nutrição de Plantas - USP/ESALQ, C.P. 9 - CEP: 13418-900 - Piracicaba, SP. \\ ${ }^{4}$ Bolsista CAPES. \\ *Autor correspondente <jdckiehl@esalq.usp.br>
}

\begin{abstract}
RESUMO: Diante dos riscos ambientais relacionados ao uso agrícola de lodo de esgoto contendo metais foi realizado um experimento, com o objetivo de avaliar o efeito do lodo, combinado ou não com $\mathrm{P}, \mathrm{K}$ e PK, sobre o acúmulo de metais na parte aérea das plantas e comparar a eficiência de extratores na fitodisponibilidade de metais. Amostras da camada de $0-20 \mathrm{~cm}$ de um Argissolo Vermelho Amarelo eutrófico foram transferidas para vasos e tratadas com doses equivalentes a 0,10, 20, 30, 40 e $50 \mathrm{Mg} \mathrm{ha}^{-1}$ de lodo (base seca). Após 30 dias de incubação, determinou-se os teores de $\mathrm{Fe}, \mathrm{Mn}, \mathrm{Cu}, \mathrm{Zn}, \mathrm{Cd}, \mathrm{Ni}, \mathrm{Cr}$ e $\mathrm{Pb}$ (extraídos por $\mathrm{HCl} 0,1 \mathrm{~mol} \mathrm{~L}^{-1}$, Mehlich-3 e DTPA pH 7,3), procedeu-se aplicação de duas doses de $\mathrm{P}\left(0\right.$ e $\left.100 \mathrm{mg} \mathrm{kg}^{-1}\right)$ e duas dose de $\mathrm{K}(0$ e $100 \mathrm{mg} \mathrm{kg}^{-1}$ ). Cultivaram-se plantas de milho (Zea mays L.) durante 50 dias, coletando-se a parte aérea para determinação de metais. A aplicação de lodo aumentou o teor de $\mathrm{Fe}, \mathrm{Mn}, \mathrm{Zn}$ e Cu no solo e o acúmulo pelas plantas, enquanto a complementação do resíduo com potássio aumentou a produção de matéria seca e diminuiu o acúmulo de $\mathrm{Zn}$. A redução do $\mathrm{pH}$ e o $\mathrm{Mn}$ nativo do solo influenciaram no aumento da disponibilidade $\mathrm{Mn}$. Todos os extratores foram eficientes para predição da fitodisponibilidade de Zn e Cu. Para o Mn e Fe, os mais eficientes foram: $\mathrm{HCl}$ e Mehlich-3, $\mathrm{HCl}$ e DTPA, respectivamente. Para os demais metais, os extratores se mostraram pouco eficientes.
\end{abstract}

Palavras-chave: milho, DTPA, Mehlich-3, $\mathrm{HCl}$

\section{EXTRACTION AND BIOAVAILABILITY OF HEAVY METALS IN RESPONSE TO THE ADDITION OF SEWAGE SLUDGE TO THE SOIL}

\begin{abstract}
To evaluate environmental risk of sludge application in agriculture, a greenhouse experiment was carried out in Piracicaba, SP, Brazil, to compare the efficiency of extractors in the availability of heavy metals to plants, in soil treated with sewage sludge. The effect of the sludge was also evaluated combined or not with $\mathrm{P}, \mathrm{K}$ and $\mathrm{PK}$, on the accumulation of metals in the shoot. Soil samples, collected from the $0-20 \mathrm{~cm}$ layer of a Typic Hapludalf were placed in pots and mixed with 0, 10, 20, 30, 40 and $50 \mathrm{Mg}^{-1}$ (dry-basis) of sludge. Thirty days after incubation, soil samples were taken from each pot for determination of $\mathrm{Fe}, \mathrm{Mn}, \mathrm{Cu}$, $\mathrm{Zn}, \mathrm{Cd}, \mathrm{Ni}, \mathrm{Cr}$ and $\mathrm{Pb}$ (extracted by $\mathrm{HCl} 0.1 \mathrm{~mol} \mathrm{~L}^{-1}$, Mehlich-3 and DTPA pH 7.3). Two Rates of phosphate as were applied ( 0 and $100 \mathrm{Mg} \mathrm{ha}^{-1}$ ) as well as two rates of potassium ( 0 and $\left.100 \mathrm{Mg} \mathrm{ha}^{-1}\right)$. Maize (Zea mays L.) plants were grown for 50 days, collected and submitted to heavy metal determination. The application of sewage sludge increased the amounts of $\mathrm{Fe}, \mathrm{Mn}, \mathrm{Cu}$ and $\mathrm{Zn}$ accumulated in plants, whereas the addition of potassium increased dry matter yield and decreased the accumulation of $\mathrm{Zn}$. The $\mathrm{pH}$ reduction and soil native metal influenced the availability of $\mathrm{Mn}$. All extractors were efficient in predicting the $\mathrm{Zn}$ and $\mathrm{Cu}$ availability to plants. For $\mathrm{Mn}$ and $\mathrm{Fe}$ the most efficient solutions were: $\mathrm{HCl}$ and $\mathrm{Mehlich}-3, \mathrm{HCl}$ and DTPA, respectively. For the other metals, none of the extractors was efficient.
\end{abstract}

Key words: maize, DTPA, Mehlich-3, $\mathrm{HCl}$

\section{INTRODUÇÃO}

Diversos trabalhos têm demonstrado que a aplicação de lodo de esgoto promove o aumento da concentração de metais no solo (Chang et al., 1987; Mulchi et al., 1991; Hooda \& Alloway, 1993). No entanto, devido ao comportamento desses elementos no solo, ainda existem dúvidas no que diz respeito à absorção dos metais pesados pelas plantas e à possibilidade desses elementos alcançarem concentrações fitotóxicas nos solos ou nas plantas.

A contaminação causada por metais pesados nos solos tratados com lodo de esgoto tem sido avaliada pela concentração total desses metais no solo. No entanto, o fato do metal pesado estar presente no solo não significa que esteja numa forma prontamente assimilável pelas plantas, podendo permanecer por longos períodos sem ser absorvido em quantidades tóxicas. Estudos têm demonstrado não haver correlação entre o teor total de metais pesados no solo e sua fitotoxicidade (Egreja Filho, 1993). Portanto, para se conhecer a contaminação, em termos dos efeitos sobre as plantas e a cadeia alimentar, é necessário determinar as concentrações fitodisponíveis desses metais (Leschber et al., 1985).

${ }^{1}$ Parte da Tese de Doutorado da primeira autora apresentada à USP/ESALQ - Piracicaba, SP. 
Entre os extratores encontrados na literatura, para estudar a fitodisponibilidade em áreas que recebem lodo de esgoto, pode-se destacar o ligante orgânico ou complexante DTPA e as soluções ácidas $\mathrm{HCl} 0,1 \mathrm{~mol} \mathrm{~L}^{-1}$ e Mehlich-3 (Roca \& Pomares, 1991; Bertoncini, 1997; Anjos, 1999; Oliveira, 2000). O uso de agentes complexantes decorre da sua habilidade de deslocar metais ligados a radicais orgânicos e carbonatos, extraindo com facilidade as formas lábeis dos metais, sem dissolver as não lábeis (Abreu et al., 1997). O extrator DTPA a pH 7,3, foi proposto por Lindsay \& Norvell (1978), para determinação de $\mathrm{Cu}, \mathrm{Zn}, \mathrm{Fe}$ e $\mathrm{Mn}$ em solos calcários e em solos com valores de $\mathrm{pH}$ próximos à neutralidade. Atualmente, os laboratórios de análise de solo do Estado de São Paulo têm utilizado esse extrator como método oficial para determinação de $\mathrm{Fe}, \mathrm{Cu}, \mathrm{Mn}$ e $\mathrm{Zn}$ (Cantarella et al., 1995). Em diversos trabalhos esta solução também tem sido utilizada para determinar outros metais como $\mathrm{Cd}$, $\mathrm{Ni}$ e $\mathrm{Pb}$ em solos (Mulchi et al.1991; Abreu et al., 1995; Oliveira, 2000).

$\mathrm{O}$ extrator ácido $\mathrm{HCl} 0,1 \mathrm{~mol} \mathrm{~L}^{-1}$ extrai quantidades próximas do total devido ao seu poder de dissolver, mesmo que parcialmente, as estruturas minerais que retêm metais pesados no solo. Apesar disso, muitas vezes as quantidades extraídas correlacionam-se com as absorvidas pelas plantas (Taylor et al., 1993; Roca \& Pomares, 1991).

Recentemente, o extrator Mehlich-3 foi introduzido para o uso em solos ácidos e tem despertado crescente interesse por pesquisadores, pois dentre os métodos de extração ácida, foi considerado o mais apropriado e o mais econômico para a análise de terra em laboratório devido à sua capacidade de extrair do solo, simultaneamente, vários nutrientes $(\mathrm{P}, \mathrm{K}, \mathrm{Ca}, \mathrm{Mg}$, $\mathrm{Na}, \mathrm{B}, \mathrm{Cu}, \mathrm{Fe}, \mathrm{Mg}$ e Zn) (Tran et al., 1990). Este método foi adaptado a partir do extrator Mehlich-2 pela associação do princípio da quelação (EDTA), com a acidez para melhorar a eficiência de extração dos micronutrientes. A adição de EDTA aumentou as extrações de $\mathrm{Cu}$ em $170 \%$, a de $\mathrm{Mn}$ em $50 \%$ e a de $\mathrm{Zn}$ em $25 \%$ (Mehlich, 1984). As vantagens do uso de Mehlich-3 para análise de terra em laboratório, são permitir a determinação simultânea de vários nutrientes e exigir menor tempo de agitação (5 $\mathrm{min})$, quando comparado com o método DTPA a pH 7,3 (2 horas).

Esse trabalho teve o objetivo de avaliar, em casade-vegetação, os efeitos de doses de lodo de esgoto, combinadas ou não com adubação de $\mathrm{P}, \mathrm{K}$ e PK no acúmlo de metais em plantas de milho e selecionar extratores para a fitodisponibilidade desses metais.

\section{MATERIAL E MÉTODOS}

O trabalho foi desenvolvido em casa-devegetação, do Departamento de Solos e Nutrição de Plantas da ESALQ/USP, utilizando amostras de terra, coletadas da camada $0-20 \mathrm{~cm}$, de um Argissolo Vermelho
Amarelo eutrófico da região de Piracicaba/SP. A análise de formas totais revelou $87,7 \mathrm{~g} \mathrm{~kg}^{-1}$ de $\mathrm{Al}_{2} \mathrm{O}_{3}, 63,4 \mathrm{~g} \mathrm{~kg}^{-1}$ de $\mathrm{Fe}_{2} \mathrm{O}_{3}$ e $102 \mathrm{~g} \mathrm{~kg}^{-1}$ de $\mathrm{SiO}_{2}$, obtidos através de ataque sulfúrico (Camargo et al., 1986). As demais características químicas encontram-se na Tabela 1. Quanto à caracterização física, o solo apresentou densidade de $1,47 \mathrm{~g} \mathrm{~cm}^{-3}$ e a seguinte composição granulométrica: $400 \mathrm{~g} \mathrm{~kg}^{-1}$ de argila, $200 \mathrm{~g} \mathrm{~kg}^{-1}$ de silte $\mathrm{e}$ $400 \mathrm{~g} \mathrm{~kg}^{-1}$ de areia, determinada pelo método do densímetro (Embrapa, 1997).

O lodo utilizado, era proveniente de digestão anaeróbia (sem tratamento com cal) produzido na Estação de Tratamento das Águas do Ribeirão do Piracicamirim do Município de Piracicaba, SP, cuja caracterização química encontra-se na Tabela 1.

O experimento seguiu o delineamento inteiramente casualizado, sendo as parcelas compostas por vasos com capacidade de $5 \mathrm{~L}$, sem perfuração na parte inferior para evitar a lixiviação de elementos, distribuídas aleatoriamente na bancada. Para os metais no solo os tratamentos constituíram de seis doses de lodo de esgoto, equivalentes a 0,10,20,30, 40 e $50 \mathrm{Mg}$ ha $^{-1}$ (base seca), com 16 repetições, totalizando 96 parcelas. Para as plantas os tratamentos em arranjo fatorial $6 \times 2 \times 2$ foram constituídos pelas seis doses de lodo de esgoto somadas a duas doses de superfosfato simples ( 0 e $100 \mathrm{mg} \mathrm{kg}^{-1}$ de $\mathrm{P}$ ) e duas doses de cloreto de potássio $\left(0\right.$ e $100 \mathrm{mg} \mathrm{kg}^{-1}$ de $\mathrm{K}$ ), resultando em 24 tratamentos, e 4 repetições, totalizando 96 parcelas.

Quantidades de $3 \mathrm{~kg}$ de terra seca ao ar, passadas em peneira de $5 \mathrm{~mm}$ de abertura de malha, foram transferidas para vasos e tratadas com doses de lodo, equivalentes a $0,10,20,30,40,50 \mathrm{Mg} \mathrm{ha}^{-1}$ (base seca) e uma mistura de $\mathrm{CaCO}_{3}$ e $\mathrm{MgCO}_{3}$ p.a (proporção de 4 partes de $\mathrm{Ca}+1$ parte de $\mathrm{Mg}$ ) para elevar a saturação por bases a $70 \%$. As quantidades de água deionizada adicionadas foram correspondentes a $70 \%$ da capacidade de retenção de água do solo. As terras foram incubadas por 30 dias nos próprios vasos, em temperatura ambiente, repondo-se a água evaporada quando necessário. Cessado o período de incubação, retiraram-se amostras de terra de cada vaso, que foram secas em estufa e passadas em peneira de $2 \mathrm{~mm}$ de abertura de malha, para análise caracterização química das amostras tratadas com o resíduo (Tabela 2) e determinação dos teores solúveis de $\mathrm{Cd}, \mathrm{Cr}, \mathrm{Cu}, \mathrm{Fe}, \mathrm{Ni}$, $\mathrm{Pb}$ e $\mathrm{Zn}$ através dos extratores $\mathrm{HCl} 0,01 \mathrm{~mol} \mathrm{~L}^{-1}$ (Sauerbeck \& Styperek, 1985), Mehlich-3 (Mehlich, 1984), DTPA a pH 7,0 (Lindsay \& Norvell, 1978). As quantidades de metais presentes nos extratos foram determinadas por espectrofotometria de absorção atômica com chama convencional.

Após o período de incubação das amostras, realizaram-se as adubações fosfatada $(3,82 \mathrm{~g}$ de superfofato simples por vaso) e potássica $(0,6 \mathrm{~g}$ de cloreto de potássio por vaso). Após 24 horas foram semeadas 10 sementes de milho, híbrido AGX-7393, por 
vaso e aos sete dias da germinação foi feito o desbaste, deixando-se cinco plantas por vaso. Aos 28 dias da germinação das plantas foi aplicada a adubação nitrogenada ( $300 \mathrm{mg}$ de $\mathrm{N}$ por vaso) na forma de uréia, diluída em água, devido ao aparecimento de sintomas visuais de deficiência de N. A irrigação foi feita colocando-se água suficiente para proporcionar ao solo umidade equivalente a $70 \%$ da capacidade de retenção de água, sendo o monitoramento da umidade do solo realizado pela pesagem dos vasos. Semanalmente realizou-se o rodízio dos vasos, visando minimizar o efeito das variações de ambiente a que estavam expostos. Quando as plantas atingiram 50 dias foi coletada a parte aérea (folhas + colmos), que foi seca em estufa a $60^{\circ} \mathrm{C}$ até massa constante, pesada e moída para a análise de $\mathrm{Cd}, \mathrm{Cr}, \mathrm{Cu}, \mathrm{Fe}, \mathrm{Ni}, \mathrm{Pb}$ e $\mathrm{Zn}$ através de digestão nitroperclórica (Sarruge \& Haag, 1974). Tendo em vista que alguns metais pesados freqüentemente se apresentam em concentrações extremamente baixas, digeriram-se $1000 \mathrm{mg}$ do material com recuperação do extrato em volume de $15 \mathrm{~mL}$. A determinação analítica dos metais nas plantas foi realizada por espectrofotometria de absorção atômica com chama convencional.

Para avaliação dos extratores, os resultados dos teores de $\mathrm{Fe}, \mathrm{Mn}, \mathrm{Cu}$ e $\mathrm{Zn}$ do solo foram submetidos à

Tabela 1 - Caracterização química da amostra de lodo de esgoto e do solo utilizados no experimento.

\begin{tabular}{|c|c|c|}
\hline Característica & Lodo $^{1}$ & Solo $^{2}$ \\
\hline Teor de água & $738 \mathrm{~g} \mathrm{~kg}^{-1}$ & - \\
\hline $\mathrm{pH}\left(\mathrm{CaCl}_{2}\right)$ & 6,3 & 4,5 \\
\hline Matéria Orgânica & $523,60 \mathrm{~g} \mathrm{~kg}^{-1}$ & $17 \mathrm{~g} \mathrm{dm}^{-3}$ \\
\hline $\mathrm{N}$ & $29,10 \mathrm{~g} \mathrm{~kg}^{-1}$ & - \\
\hline $\mathrm{P}$ & $6,02 \mathrm{~g} \mathrm{~kg}^{-1}$ & $13 \mathrm{mg} \mathrm{dm}^{-3}$ \\
\hline $\mathrm{K}$ & $1,30 \mathrm{~g} \mathrm{~kg}^{-1}$ & 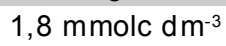 \\
\hline $\mathrm{Ca}$ & $20,80 \mathrm{~g} \mathrm{~kg}^{-1}$ & $36 \mathrm{mmolc} \mathrm{dm}^{-3}$ \\
\hline $\mathrm{Mg}$ & $3,50 \mathrm{~g} \mathrm{~kg}^{-1}$ & $20 \mathrm{mmolc} \mathrm{dm}^{-3}$ \\
\hline S & $8,30 \mathrm{~g} \mathrm{~kg}^{-1}$ & $32 \mathrm{mg} \mathrm{dm}^{-3}$ \\
\hline Fe total & $30295,00 \mathrm{mg} \mathrm{kg}^{-1}$ & - \\
\hline Mn total & $173,00 \mathrm{mg} \mathrm{kg}^{-1}$ & $536 \mathrm{mg} \mathrm{kg}^{-1}$ \\
\hline Cu total & $192,94 \mathrm{mg} \mathrm{kg}^{-1}$ & $26 \mathrm{mg} \mathrm{kg}^{-1}$ \\
\hline Zn total & $943,41 \mathrm{mg} \mathrm{kg}^{-1}$ & $32 \mathrm{mg} \mathrm{kg}^{-1}$ \\
\hline Cd total & $<0,02 \mathrm{mg} \mathrm{kg}^{-1 *}$ & $<19 \mathrm{mg} \mathrm{kg}^{-1 *}$ \\
\hline Cr total & $207,20 \mathrm{mg} \mathrm{kg}^{-1}$ & $12 \mathrm{mg} \mathrm{kg}^{-1}$ \\
\hline $\mathrm{Ni}$ total & $<0,01 \mathrm{mg} \mathrm{kg}^{-1 *}$ & $<8 \mathrm{mg} \mathrm{kg}^{-1} *$ \\
\hline $\mathrm{Pb}$ total & $107,56 \mathrm{mg} \mathrm{kg}^{-1}$ & $80 \mathrm{mg} \mathrm{kg}^{-1}$ \\
\hline $\mathrm{H}+\mathrm{Al}$ & - & $42 \mathrm{mmolc} \mathrm{dm}^{-3}$ \\
\hline Saturação por base (V) & - & $58 \%$ \\
\hline
\end{tabular}

${ }^{1}$ Resultados expressos em material seco a $65^{\circ} \mathrm{C}$. Teores totais de $\mathrm{Cu}, \mathrm{Fe}, \mathrm{Zn}, \mathrm{Mn}, \mathrm{Cd}, \mathrm{Pb}, \mathrm{Ni}, \mathrm{Cr}$ extraídos por digestão nitro-perclórica (Sarruge \& Haag, 1974). Para as demais características utilizou-se metodologia descrita por Kiehl (1985).

${ }^{2}$ Teores totais de $\mathrm{Mn}, \mathrm{Cu}, \mathrm{Zn}, \mathrm{Cr}, \mathrm{Ni}, \mathrm{Pb}$ e Cd extraídas por digestão com $\mathrm{HNO}_{3}+\mathrm{HClO}_{4}+\mathrm{HF}$ (Soltanpour et al., 1982). Para as demais característica utilizou-se metodologia descrita por Raij \& Quaggio (1983).

*Elemento encontrado abaixo do limite de determinação do método analítico utilizado.

- Característica não determinada. análise de variância considerando-se os tratamentos em arranjo fatorial envolvendo seis doses de lodo e três métodos de extração. Os métodos de extração foram comparados pelo teste de Tukey a $5 \%$. O efeito das doses de lodo foi avaliados por meio de regressões polinomiais. Também foram realizadas correlações lineares entre resultados obtidos pelos próprios extratores.

Para a avaliação de produção de matéria seca e metais acumulados nas plantas, realizou-se a análise de variância seguindo o delineamento inteiramente casualizado, com os tratamentos doses de lodo, doses de $\mathrm{P}$ e doses de $\mathrm{K}$, em arranjo fatorial $6 \times 2 \times 2$. O efeito das doses de lodo foi avaliado por meio de regressões polinomiais.

A fitodisponibilidade de metais foi avaliada através da correlação linear entre os resultados obtidos pelos extratores e a quantidade de metais acumuladas pela parte aérea das plantas.

\section{RESULTADOS E DISCUSSÃO}

\section{Avaliação de Extratores}

Os teores de $\mathrm{Cd}, \mathrm{Cr}, \mathrm{Pb}$ e $\mathrm{Ni}$ eventualmente

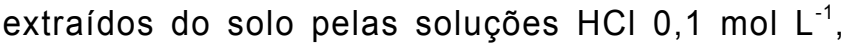
Mehlich-3 e DTPA apresentaram-se, para todos os tratamentos, abaixo do limite de determinação do método analítico utilizado. Dessa forma, para o extrator $\mathrm{HCl} 0,1$ $\mathrm{mol} \mathrm{L}^{-1}$ as quantidades desses metais nos extratos foram respectivamente, $<1,0,<0,6,<4,1 \mathrm{e}<0,4 \mathrm{mg} \mathrm{kg}^{-1}$. Para o Mehlich-3 as quantidades foram $<2,0,<1,5$, $<8,0$. e $<0,5 \mathrm{mg} \mathrm{kg}^{-1}$ ao passo que para o DTPA as quantidades foram $<0,4,<0,3,<2,0 \mathrm{e}<0,2 \mathrm{mg} \mathrm{kg}^{-1}$.

Oliveira (2000), em condições de campo, avaliando a fitodisponibilidade de metais pesados por diferentes extratores, em Latossolo Vermelho Amarelo tratado com 33, 66 e $99 \mathrm{Mg} \mathrm{ha}^{-1}$ (base seca) de lodo no primeiro ano e 37, 74 e $110 \mathrm{Mg} \mathrm{ha}^{-1}$ (base seca) no segundo ano, constatou que os teores de $\mathrm{Cd}$, $\mathrm{Cr}$ e $\mathrm{Pb}$, avaliados pelos extratores $\mathrm{HCl} 0,1 \mathrm{~mol} \mathrm{~L}^{-1}$, Mehlich-3 e DTPA, estiveram abaixo do limite de determinação do método analítico utilizado. Para o Ni, o autor constatou remoção média de $8 \%, 28 \%$ e $23 \%$, respectivamente, para os extratores $\mathrm{HCl} 0,1 \mathrm{~mol} \mathrm{~L}^{-1}$, Mehlich-3 e DTPA.

Tabela 2 - Características químicas das amostras de terra após período de incubação.

\begin{tabular}{lcccc}
\hline Dose de lodo & $\mathrm{pH}$ & $\mathrm{MO}$ & $\mathrm{CTC}^{*}$ & $\mathrm{~V}$ \\
\hline $\mathrm{Mg} \mathrm{ha}^{-1}$ & & $\mathrm{~g} \mathrm{dm}^{-3}$ & $\mathrm{mmol}_{\mathrm{c}} \mathrm{dm}^{-3}$ & $\%$ \\
0 & 5,1 & 17 & 99,9 & 70 \\
10 & 5,0 & 17 & 110,8 & 70 \\
20 & 4,8 & 19 & 117,0 & 70 \\
30 & 4,5 & 21 & 129,1 & 70 \\
40 & 4,7 & 23 & 134,4 & 70 \\
50 & 4,7 & 23 & 156,6 & 73 \\
\hline
\end{tabular}

${ }^{1}$ Análise segundo metodologia descrita por Raij \& Quaggio (1983). *Estimada (SB + H+Al). 
As quantidades de $\mathrm{Fe}, \mathrm{Mn}$, $\mathrm{Cu}$ e $\mathrm{Zn}$ obtidas pelos extratores, das amostras tratadas com doses crescentes de lodo, são apresentadas na Tabela 4. Considerando as quantidades desses metais adicionadas ao solo, via lodo de esgoto (Tabela 3), é possível estimar que a quantidade média de ferro extraída foi de $48 \%, 13 \%$ e $5 \%$, respectivamente, para os extratores $\mathrm{HCl} 0,1$ mol L ${ }^{-1}$, Mehlich-3 e DTPA; para o cobre, tais valores foram de $52 \%, 37 \%$ e $26 \%$, e para o zinco, de $80 \%, 59 \%$ e $43 \%$, respectivamente. As correlações lineares entre a quantidade de $\mathrm{Fe}$ e $\mathrm{Zn}$ extraída pelos extratores $\mathrm{HCl} 0,1 \mathrm{~mol} \mathrm{~L}^{-1}$, Mehlich-3 e DTPA apresentadas pelas amostras de solo foram altamente significativas. Para o $\mathrm{Cu}$, as correlações também foram altamente significativas, embora, o extrator $\mathrm{HCl} 0,1 \mathrm{~mol} \mathrm{~L}^{-1}$ tenha apresentado correlação quadrática com tendência decrescente em seus valores, em função do aumento das doses de lodo aplicadas.

As soluções $\mathrm{HCl} 0,1 \mathrm{~mol} \mathrm{~L}^{-1}$, Mehlich-3 e DTPA extraíram, respectivamente, $1407 \%, 619 \%$ e $133 \%$ acima das quantidades de manganês adicionadas via resíduo. Esse comportamento está, provavelmente, relacionado à redução do pH do solo (Tabela 1). As correlações lineares entre a quantidade de manganês extraída pelos extratores $\mathrm{HCl} 0,1 \mathrm{~mol} \mathrm{~L}^{-1}$, Mehlich-3 e DTPA apresentadas pelas amostras de solo foram altamente significativas $\left(r=-0,704^{* *}, r=-0,700^{* *}, r=-0,702^{* *}\right.$, respectivamente). A solubilidade do $\mathrm{Mn}$ no solo é altamente dependente do $\mathrm{pH}$ e do potencial redox (Borkert, 1991). Em solos bem drenados, a solubilidade do Mn aumenta com o aumento da acidez do solo, sendo que a concentração de $\mathrm{Mn}$ na solução pode aumentar em 100 vezes para cada unidade de decréscimo de $\mathrm{pH}$ (Tisdale et al., 1985).

Trabalhando em solos Neossolo Quartzarênico (Areia Quartzosa) e Latossolo Vermelho eutroférrico (Latossolo Roxo), com níveis de $\mathrm{pH}$ ajustados para 3,9 e 4,9, e posteriormente tratados com $0 ; 13,5 ; 29,7$ e 40,5 $\mathrm{Mg} \mathrm{ha}^{-1}$ (base seca) de lodo de esgoto, Oliveira (1995) verificou que as soluções $\mathrm{HCl} 0,1 \mathrm{~mol} \mathrm{~L}^{-1}$ e DTPA extraíram mais $\mathrm{Mn}$ do que as quantidades adicionadas ao solo via lodo de esgoto.

As soluções ácidas (extratores $\mathrm{HCl} 0,1 \mathrm{~mol} \mathrm{~L}^{-1} \mathrm{e}$ Mehlich-3) extraíram, nos tratamentos que receberam doses do resíduo, quantidades superiores de $\mathrm{Fe}, \mathrm{Mn}, \mathrm{Cu}$ e $\mathrm{Zn}$ em relação à solução quelante (DTPA), apresentando a seguinte ordem de extração: $\mathrm{HCl} 0,1 \mathrm{~mol}$ $\mathrm{L}^{-1}>$ Mehlich-3 > DTPA. A exceção foi para o $\mathrm{Cu}$, nos tratamentos que receberam doses de 10 e $50 \mathrm{Mg} \mathrm{ha}^{-1}$ de lodo, onde Mehlich-3 e DTPA não diferiram na capacidade de extração. Esses resultados são explicados pelo fato das soluções ácidas extraírem quantidades próximas do total, devido ao seu potencial em dissolver certas estruturas que retém metais no solo (Abreu et al., 1995). De forma concordante, Mulchi et al. (1991), trabalhando com solos franco siltoso e franco arenoso, tratados com diferentes tipos de lodo, verificaram que os extratores ácidos (Mehlich-1 e Mehlich-3) foram superiores na extração dos metais $\mathrm{Fe}$, $\mathrm{Mn}$ e $\mathrm{Zn}$, em relação ao extrator DTPA, para todas as doses e tipos de lodo utilizados. Quanto ao $\mathrm{Cu}$, aqueles autores constataram que, para os solos tratados com lodo digerido desidratado e lodo digerido alcalinizado, os extratores ácidos foram superiores ao DTPA. Para os solos com composto de lodo alcalinizado e lodo tratado com Fe e submetido a aquecimento, o extrator DTPA foi superior aos extratores ácidos.

No tratamento sem aplicação de lodo (Tabela 4), o Mehlich-3 extraiu maior quantidade de $\mathrm{Fe}$ e $\mathrm{Zn}$ em relação aos extratores $\mathrm{HCl} 0,1 \mathrm{~mol} \mathrm{~L}^{-1}$ e DTPA. Para o $\mathrm{Mn}$, a ordem de extração foi $\mathrm{HCl}>$ Mehlich-3 > DTPA. No entanto, as quantidades extraídas de $\mathrm{Cu}$, pelos diferentes métodos, foram iguais.

Bertoncini (1997) constatou que em Neossolo Quartzarênico (Areia Quartzosa) e Latossolo Vermelho Amarelo, os extratores $\mathrm{HCl} \mathrm{0,1} \mathrm{mol} \mathrm{L}^{-1}$, Mehlich-3 e DTPA não diferiram na determinação dos teores solúveis de $\mathrm{Cu}$, enquanto que para Latossolo Vermelho Escuro o extrator $\mathrm{HCl} 0,1 \mathrm{~mol} \mathrm{~L}^{-1}$ suplantou o Mehlich-3 e DTPA. Para o $\mathrm{Zn}$, a autora verificou que três métodos estudados não diferiram na extração do elemento nos três solos estudados.

Os teores de cobre aumentaram, em média, de $29 \%$ a $170 \%, 15 \%$ a $103 \%$ e $18 \%$ a $88 \%$, respectivamente, para os extratores $\mathrm{HCl} 0,1 \mathrm{~mol} \mathrm{~L}^{-1}$, Mehlich-3 e DTPA. Para o manganês, os aumentos variaram de $12 \%$ a $83 \%$, de $10 \%$ a $63 \%$ e de $11 \%$ a $99 \%$, respectivamente. Aumentos nos teores disponíveis de ferro, cobre, manganês e zinco com a disposição de lodo de esgoto no solo também foram observados por Mulchi et al. (1991), Hooda \& Alloway (1993) e Hooda et al. (1997).

Tabela 3 - Quantidades de metais adicionadas às amostras de terra em função da adição das doses de lodo de esgoto.

\begin{tabular}{lcccccc}
\hline Dose de lodo & Ferro & Manganês & Crômio & Chumbo & Cobre & Zinco \\
\hline Mg ha ${ }^{-1}$ & - & 0,92 & 1,11 & 0,58 & 1,03 & 5,05 \\
10 & 162,08 & 1,85 & 2,22 & 1,15 & 2,06 & 10,09 \\
20 & 324,16 & 2,78 & 3,33 & 1,73 & 3,10 & 15,14 \\
30 & 486,23 & 3,70 & 4,43 & 2,30 & 4,13 & 20,19 \\
40 & 648,31 & 4,63 & 5,54 & 2,88 & 5,16 & 25,24 \\
50 & 810,39 & 2,78 & 3,33 & 1,73 & 3,10 & 15,14 \\
\hline
\end{tabular}


Tabela 4 - Teores de ferro, manganês, cobre e zinco obtidos por diferentes extratores, em função da aplicação de doses de lodo de esgoto

\begin{tabular}{|c|c|c|c|}
\hline Dose de Lodo de & $\mathrm{HCl} 0,1 \mathrm{~mol} \mathrm{~L}^{-1}$ & Mehlich-3 & DTPA a $\mathrm{pH} 7,0$ \\
\hline $\mathrm{Mg} \mathrm{ha}^{-1}$ & \multicolumn{3}{|c|}{ Ferro (mg kg-1) } \\
\hline 0 & $82,25 \mathrm{~b}$ & $105,00 \mathrm{a}$ & $30,37 \mathrm{c}$ \\
\hline 10 & $163,25 a$ & $126,75 \mathrm{~b}$ & $38,52 \mathrm{c}$ \\
\hline 20 & $233,00 \mathrm{a}$ & $142,37 b$ & $44,93 \mathrm{c}$ \\
\hline 30 & $297,50 \mathrm{a}$ & $157,75 b$ & $52,90 \mathrm{c}$ \\
\hline 40 & 396,75 a & $201,00 \mathrm{~b}$ & $68,28 \mathrm{c}$ \\
\hline 50 & $489,25 \mathrm{a}$ & $222,50 \mathrm{~b}$ & $78,10 \mathrm{c}$ \\
\hline Média & 277,00 & 159,23 & 52,18 \\
\hline Modelo & $Y=8 x+77$ & $Y=2,359 x+100,25$ & $Y=0,959 x+28,19$ \\
\hline \multirow[t]{2}{*}{ Termo de regressão $\left(R^{2}\right)$} & $0,995^{\star *}$ & $0,971^{\text {** }}$ & $0,979^{* *}$ \\
\hline & \multicolumn{3}{|c|}{ Manganês (mg kg-1) } \\
\hline 0 & $96,00 \mathrm{a}$ & $60,25 b$ & $13,25 \mathrm{c}$ \\
\hline 10 & 107,94 a & $66,60 \mathrm{~b}$ & $14,48 \mathrm{c}$ \\
\hline 20 & $123,50 \mathrm{a}$ & $73,93 \mathrm{~b}$ & $17,90 \mathrm{c}$ \\
\hline 30 & $134,25 \mathrm{a}$ & $78,02 \mathrm{~b}$ & $19,45 \mathrm{c}$ \\
\hline 40 & $156,75 \mathrm{a}$ & $86,94 \mathrm{~b}$ & $22,82 \mathrm{c}$ \\
\hline 50 & $176,13 \mathrm{a}$ & $98,18 \mathrm{~b}$ & $26,80 \mathrm{c}$ \\
\hline Média & 132,43 & 77,32 & 19,12 \\
\hline Modelo & $Y=1,594 x+92,583$ & $Y=0,7279 x+59,123$ & $Y=0,2695 x+12,38$ \\
\hline \multirow[t]{2}{*}{ Termo de regressão $\left(R^{2}\right)$} & $0,985^{\star *}$ & $0,980^{* *}$ & $0,976^{\star *}$ \\
\hline & \multicolumn{3}{|c|}{ Cobre $\left(\mathrm{mg} \mathrm{kg}^{-1}\right)$} \\
\hline 0 & $2,00 \mathrm{a}$ & $1,70 \mathrm{a}$ & $1,65 \mathrm{a}$ \\
\hline 10 & $2,58 \mathrm{a}$ & $2,00 \mathrm{~b}$ & $1,95 \mathrm{~b}$ \\
\hline 20 & $2,70 \mathrm{a}$ & $2,60 \mathrm{a}$ & $2,13 b$ \\
\hline 30 & $3,43 a$ & $2,89 \mathrm{~b}$ & $2,33 \mathrm{c}$ \\
\hline 40 & 4,35 a & $3,20 \mathrm{~b}$ & $2,68 \mathrm{c}$ \\
\hline 50 & $5,40 a$ & $3,53 b$ & $3,10 b$ \\
\hline Média & 3,41 & 2,65 & 2,31 \\
\hline Modelo & $Y=0,001 x^{2}+0,016 x+2,09$ & $Y=0,0373 x+1,722$ & $Y=0,028 x+1,62$ \\
\hline \multirow[t]{2}{*}{ Termo de regressão $\left(R^{2}\right)$} & $0,991^{* *}$ & $0,988^{* *}$ & $0,977^{* *}$ \\
\hline & \multicolumn{3}{|c|}{ Zinco (mg kg-1) } \\
\hline 0 & $1,73 a b$ & $2,18 a$ & $1,13 \mathrm{~b}$ \\
\hline 10 & $5,70 \mathrm{a}$ & 4,88 a & $3,00 \mathrm{~b}$ \\
\hline 20 & $8,80 \mathrm{a}$ & $7,65 b$ & $5,15 \mathrm{c}$ \\
\hline 30 & $14,00 \mathrm{a}$ & $11,05 b$ & $7,53 \mathrm{c}$ \\
\hline 40 & $18,50 \mathrm{a}$ & $15,35 b$ & $10,48 \mathrm{c}$ \\
\hline 50 & $24,31 \mathrm{a}$ & $18,43 b$ & $13,08 \mathrm{c}$ \\
\hline Média & 12,17 & 9,92 & 6,73 \\
\hline Modelo & $y=0,447 x+0,995$ & $y=0,332 x+1,633$ & $y=0,242 x+0,688$ \\
\hline Termo de regressão $\left(R^{2}\right)$ & $0,991^{* *}$ & $0,994^{* *}$ & $0,994^{\star *}$ \\
\hline
\end{tabular}

Médias seguidas de letras distintas na horizontal diferem pelo teste de Tukey a $5 \%$.

${ }^{* *}$ Indica que as regressões foram significativas a $P \leq 0,01$.

Embora os extratores tenham diferido na capacidade de extração dos metais, houve correlações significativas entre todos os métodos para os elementos $\mathrm{Fe}, \mathrm{Mn}, \mathrm{Cu}$ e $\mathrm{Zn}$ (Tabela 5). Portanto, todos os extratores fornecem informações semelhantes na disponibilidade desses metais em função da adição de doses do resíduo, exceto para o cobre e manganês que apresentaram baixo índice de correlação entre os extratores Mehlich-3 e DTPA e entre o $\mathrm{HCl} 0,1 \mathrm{~mol} \mathrm{~L}^{-1}$ e o Mehlich-3, respectivamente. Correlações entre os extratores $\mathrm{HCl} 0,1 \mathrm{~mol} \mathrm{~L}^{-1}$, Mehlich-3 e DTPA na determinação dos teores de $\mathrm{Cu}$ e $\mathrm{Zn}$ foram encontrados por Oliveira (2000), em Latossolo Vermelho Amarelo tratado ou não com lodo de esgoto. 
Tabela 5 - Coeficiente de correlação linear simples para os teores de $\mathrm{Fe}, \mathrm{Mn}$, Cu e $\mathrm{Zn}$ entre os extratores $\mathrm{HCl} 0,1 \mathrm{~mol} \mathrm{~L}^{-1}$ $(\mathrm{HCl})$, Mehlich-3 (M-3) e DTPA.

\begin{tabular}{|c|c|c|c|c|c|c|c|c|}
\hline \multirow[t]{2}{*}{ Extrator } & \multicolumn{2}{|c|}{ Ferro } & \multicolumn{2}{|c|}{ Manganês } & \multicolumn{2}{|c|}{ Cobre } & \multicolumn{2}{|c|}{ Zinco } \\
\hline & $\mathrm{HCl}$ & M-3 & $\mathrm{HCl}$ & M-3 & $\mathrm{HCl}$ & M-3 & $\mathrm{HCl}$ & M-3 \\
\hline DTPA & $0,94^{* *}$ & $0,93^{* *}$ & $0,81^{* *}$ & $0,66^{* *}$ & $0,95^{\text {** }}$ & $0,60^{* *}$ & $0,99^{\text {** }}$ & $0,97^{* *}$ \\
\hline M-3 & $0,95^{\text {** }}$ & - & $0,60^{* *}$ & - & $0,64^{* *}$ & - & $0,97^{\text {** }}$ & - \\
\hline
\end{tabular}

${ }^{\star \star}$ Indica que as correlações foram significativas a $P \leq 0,01$ pelo teste $\mathrm{t}$.

\section{Produção de Matéria Seca}

A produção de matéria seca das plantas aumentou com a dose de lodo, na presença ou ausência de potássio (Figura 1). Aumentos na produção de matéria seca em plantas de milho, com a aplicação de lodo, também foram observados por Oliveira (1995) e Anjos (1999). Contudo, a complementação do lodo com K proporcionou maior produção de matéria seca em relação aos tratamentos que receberam apenas lodo. Isso era esperado, uma vez que tanto o solo quanto o lodo apresentavam baixo teor desse elemento $\left(1,8 \mathrm{mmol}_{\mathrm{c}}\right.$ $\mathrm{dm}^{-3}$ e $1,3 \mathrm{~g} \mathrm{~kg}^{-1}$, respectivamente). Efeitos da complementação do lodo com $\mathrm{K}$ sobre a produção de matéria seca do sorgo granífero foram observados por Oliveira et al. (1995).

A inexistência da interação doses de lodo e doses de $P$ deve-se ao suprimento deste nutriente em quantidades adequadas via lodo, podendo-se concluir que o efeito do lodo está ligado ao suprimento do P para as plantas. Esses resultados concordam com os de Buting (1963) que afirmou que os teores de $P$ contidos no lodo são adequados para as necessidades das plantas.

\section{Acúmulo de Metais nas Plantas}

Os resultados referentes a $\mathrm{Cd}, \mathrm{Cr}, \mathrm{Ni}$ e $\mathrm{Pb}$ na parte aérea das plantas de milho não são apresentados, tendo em vista que suas concentrações estiveram abaixo do limite de determinação do método analítico empregado. Contudo, é possível assegurar que, caso as plantas tivessem absorvido tais elementos, esses teriam apresentado quantidades de $\mathrm{Cd}<1,8 \mathrm{mg}$ $\mathrm{kg}^{-1}, \mathrm{Cr}<0,60 \mathrm{mg} \mathrm{kg}^{-1}, \mathrm{Ni}<0,70 \mathrm{mg} \mathrm{kg}^{-1}$ e $\mathrm{Pb}<6,0$ $\mathrm{mg} \mathrm{kg}^{-1}$.

Oliveira (2000), avaliando a possibilidade de incrementos de metais pesados em plantas de cana-deaçúcar cultivadas em Latossolo Vermelho Amarelo tratadas com 33, 66 e $99 \mathrm{Mg} \mathrm{ha}^{-1}$ (base seca) de lodo de esgoto no primeiro ano e 37,74 e $110 \mathrm{Mg} \mathrm{ha}^{-1}$ (base seca) no segundo ano, também verificou que os possíveis incrementos de $\mathrm{Cd}, \mathrm{Cr}$, $\mathrm{Ni}$ e $\mathrm{Pb}$ nas plantas apresentaram concentrações abaixo do limite de determinação do método utilizado.

Houve efeito da aplicação das doses de lodo de esgoto no acúmulo de $\mathrm{Fe}, \mathrm{Mn}$ e Cu pelas plantas (Figura 2). A inexistência de interações entre dose de lodo, doses de fósforo e doses de potássio revela que a incorporação de lodo de esgoto no solo atua de forma predominante, no acúmulo de $\mathrm{Fe}, \mathrm{Mn}$ e $\mathrm{Cu}$ pelas plantas.

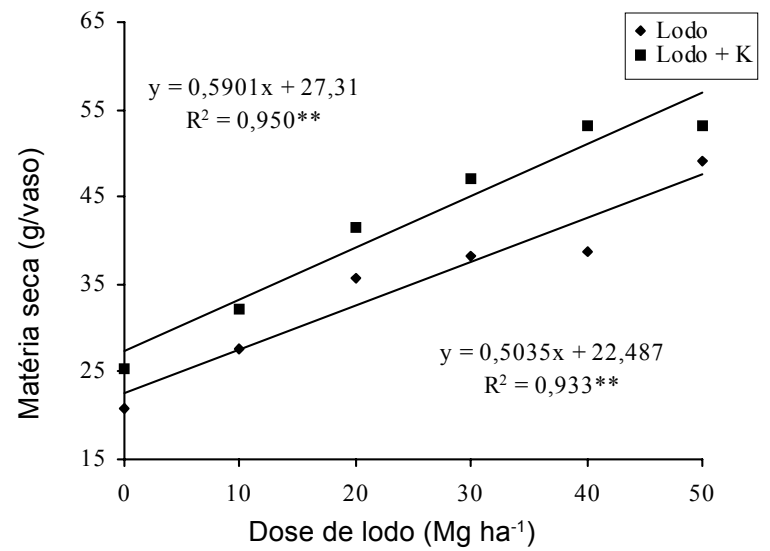

Figura 1 - Produção de matéria seca (MS) da parte aérea das plantas, em função da aplicação de lodo de esgoto.

Avaliando-se o comportamento do ferro e do cobre acumulado pelas plantas, através da análise de regressão (Figura 2), verifica-se que os dois metais apresentaram um comportamento linear crescente $\left(R^{2}=\right.$ 0,958 e $R^{2}=0,976$, respectivamente) com as doses do resíduo. Os incrementos de ferro proporcionados pela adição do resíduo variaram, da menor para a maior dose aplicada, em relação a testemunha, de $18 \%$ a $159 \%$, respectivamente. Para o cobre a variação foi de $38 \%$ a $62 \%$, respectivamente.

Quanto ao $\mathrm{Mn}$, a adição de doses de lodo promoveu um comportamento quadrático com tendência crescente $\left(R^{2}=0,958\right)$ nas quantidades acumuladas pelas plantas (Figura 2). Os incrementos de manganês proporcionados pela adição do resíduo variaram, da menor para a maior dose aplicada, de $35 \%$ a $660 \%$, respectivamente.

Para o zinco (Figura 2), houve interação entre doses de lodo e de potássio. A quantidade de zinco acumulada pelas plantas aumentou com a dose de lodo, na presença ou ausência de potássio, de forma quadrática ( $R^{2}=0,995$ e $R^{2}=0,996$, respectivamente). $\mathrm{O}$ acúmulo de zinco aumentou, da menor para a maior dose aplicada, de $18 \%$ a $159 \%$. Para o cobre, a variação foi de $38 \%$ a $62 \%$. Nos tratamentos que receberam lodo com complementação potássica as plantas apresentaram menor acúmulo de zinco, em relação aos tratamentos que receberam apenas lodo. Isso poderia ser explicado em termos de adsorção. Íons $\mathrm{Cl}^{-}$induzem a adsorção de $\mathrm{Zn}$, formando, provavelmente, um complexo estável com o zinco, principalmente a valores de $\mathrm{pH}$ mais baixos (4 4,5) (Shuman, 1986). 
Incrementos no acúmulo desses metais pelas plantas com a aplicação de lodo de esgoto são explicados pela presença desses elementos no lodo (Tabela 1). Entretanto, o solo apresentou decréscimo nos valores de $\mathrm{pH}$ com a adição de lodo (Tabela 2). A disponibilidade de metais é aumentada com a elevação da acidez do solo (Kabata-Pendias \& Pendias, 1984). Sendo assim, acredita-se que, principalmente para o Mn, onde as quantidades adicionadas foram relativamente baixas em relação aos demais metais, o aumento da acidez do solo exerceu grande influência na disponibilidade do elemento para as plantas. Por outro lado, a adição de lodo de esgoto promove aumento na absorção de ferro, manganês, cobre e zinco pelas plantas (Mulchi et al., 1991 e Anjos \& Mattiazzo, 2000), como se observou no presente trabalho.

Os teores de $\mathrm{Fe}$ e $\mathrm{Cu}$ encontrados na parte aérea das plantas, em todos os tratamentos, encontramse dentro dos intervalos considerados adequados para as plantas de milho, que são de $6-20 \mathrm{mg} \mathrm{kg}^{-1}$ e 50 $250 \mathrm{mg} \mathrm{kg}^{-1}$, respectivamente (Malavolta et al.,1997; Raij et al., 1996). Para o Mn e Zn, os teores obtidos para as duas maiores doses de lodo encontram-se acima da faixa $50-150 \mathrm{mg} \mathrm{kg}^{-1}$ e $15-50 \mathrm{mg} \mathrm{kg}^{-1}$, respectivamente,
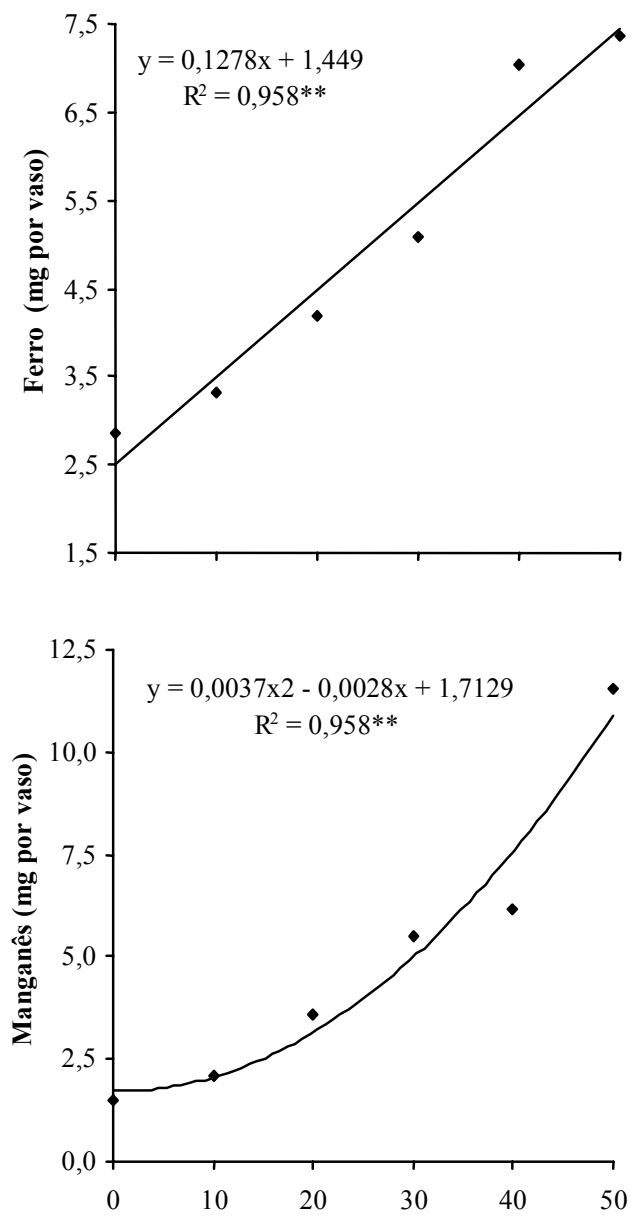

Dose de lodo $\left(\mathrm{Mg} \mathrm{ha}^{-1}\right)$ considerada adequada por Malavolta et al. (1997). Isto sugere que plantas cultivadas em solos tratados durante vários anos com lodo poderão apresentar problemas relacionados com fitotoxicidade. Observações semelhantes também foram feitas por Oliveira (1995) e Anjos \& Mattiazzo (2000).

Considerando-se as quantidades de metais adicionadas ao solo via lodo de esgoto (Tabela 3 ), as plantas removeram em média $0,3 \%, 0,9 \%$ e $39 \%$ do total de $\mathrm{Fe}$, Cu e Mn, respectivamente. Para o Zn, essas quantidades foram de $4 \%$ e $2,64 \%$, respectivamente para os tratamentos que receberam apenas lodo e nos tratamentos que receberam lodo mais adubação com $\mathrm{K}$. Entretanto, Chang et al. (1987), em amplo estudo sobre a absorção de metais pesados por plantas cultivadas em solos que receberam doses anuais de lodo, por aproximadamente 10 anos, constataram que a absorção de metais pelas plantas, de maneira geral, é inferior a $1 \%$ da quantidade adicionada via lodo de esgoto.

\section{Fitodisponibilidade de Metais}

A predição da fitodisponibilidade de metais às plantas de milho foi avaliada apenas para $\mathrm{Fe}, \mathrm{Mn}, \mathrm{Cu}$ e $\mathrm{Zn}$, tendo em vista que $\mathrm{Cd}, \mathrm{Cr}$, $\mathrm{Ni}$ e $\mathrm{Pb}$ não foram detectados nas amostras das plantas. Sendo assim, na
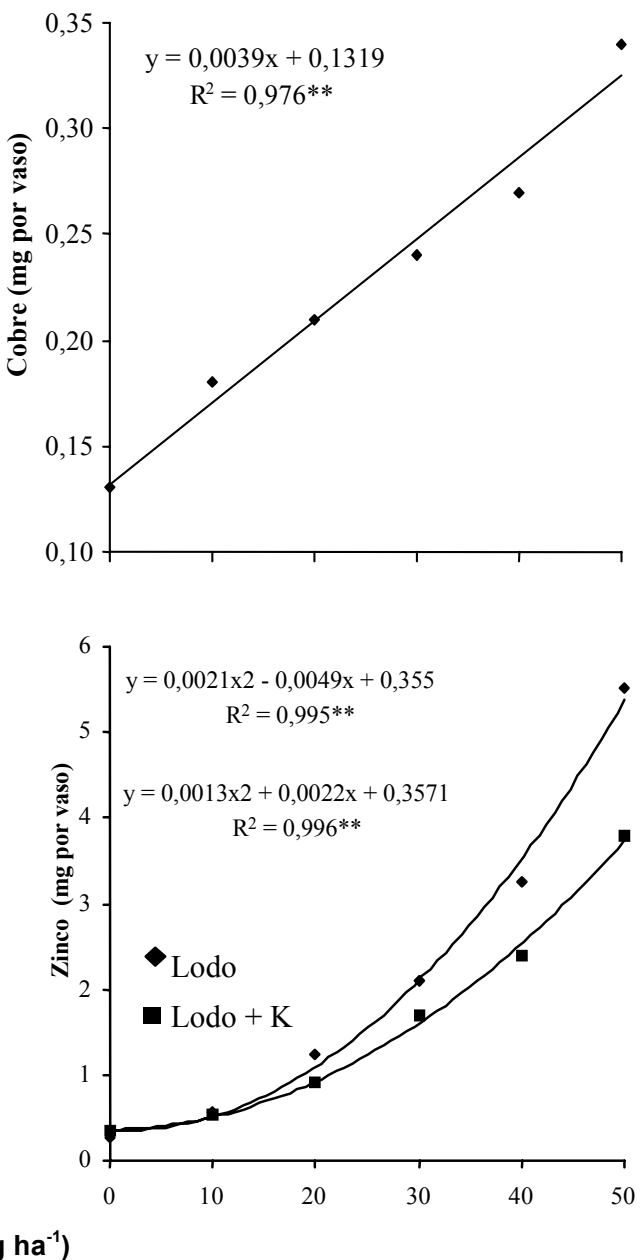

Figura 2 - Ferro, cobre, manganês e zinco acumulados pela parte aérea das plantas em função da aplicação de lodo. 
Tabela 6 - Coeficiente de correlação linear simples entre o acúmulo de metal pelas plantas e os teores extraídos do solo nos tratamentos que receberam lodo de esgoto.

\begin{tabular}{lcccc}
\hline Extrator & Ferro & Manganês & Cobre & Zinco \\
\hline $\mathrm{HCl} 0,1 \mathrm{~mol} \mathrm{~L}^{-1}$ & $0,78^{* *}$ & $0,81^{* *}$ & $0,85^{* *}$ & $0,86^{* *}$ \\
Mehlich-3 & $0,68^{* *}$ & $0,82^{* *}$ & $0,83^{* *}$ & $0,87^{* *}$ \\
DTPA & $0,72^{* *}$ & $0,65^{* *}$ & $0,87^{* *}$ & $0,89^{* *}$ \\
\hline
\end{tabular}

${ }^{* *}$ Indica que as correlações foram significativas a $P \leq 0,01$ pelo teste $\mathrm{t}$.

Tabela 6 são apresentados os coeficientes de correlação linear simples obtidos entre as quantidades totais de $\mathrm{Fe}$, $\mathrm{Mn}, \mathrm{Cu}$ e $\mathrm{Zn}$ encontrados nas plantas e os teores desses elementos extraídos do solo nos tratamentos que receberam lodo de esgoto.

Todas as correlações foram significativas a $1 \%$, destacando-se as obtidas para o $\mathrm{Zn}$ e $\mathrm{Cu}$, com todos os extratores, as obtidas para o $\mathrm{Mn}$ com $\mathrm{HCl} 0,01 \mathrm{~mol} \mathrm{~L}^{-1} \mathrm{e}$

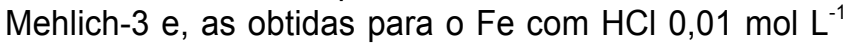
e DTPA. De forma concordante, Oliveira (2000), trabalhando em Latossolo Vermelho Amarelo cultivado com cana-de-açúcar, tratado com doses de 33, 66 e 99 $\mathrm{Mg} \mathrm{ha}^{-1}$ (base seca) de lodo no primeiro ano e $37,74 \mathrm{e}$ $110 \mathrm{Mg} \mathrm{ha}^{-1}$ (base seca) de lodo no segundo ano, observou que os extratores $\mathrm{HCl} 0,1 \mathrm{~mol} \mathrm{~L}^{-1}$, DTPA e Mehlich-3 foram eficientes na avaliação da fitodisponibilidade de $\mathrm{Zn}$, quando considerado apenas os tratamentos que receberam doses de lodo.

Anjos (1999), avaliando a eficiência dos extratores $\mathrm{HCl} 0,1 \mathrm{~mol} \mathrm{~L}^{-1}$, Mehlich-3 e DTPA na fitodisponibilidade de metais para plantas de milho cultivadas em Latossolo Vermelho Amarelo e Latossolo Vermelho eutroférrico, que receberam doses sucessivas de lodo, que totalizaram $387,89 \mathrm{Mg} \mathrm{ha}^{-1}$ (base seca), constatou que, dentre os extratores, o Mehlich-3 foi 0 mais eficiente na avaliação da disponibilidade de Zn para as plantas, nos dois solos. Porém nenhum extrator foi eficiente na avaliação da fitodisponibilidade de manganês. A ineficiência dos extratores, na predição da fitodisponibilidade de $\mathrm{Mn}$ foi associada ao fato de que, embora o teor disponível do Mn tenha aumentado no Latossolo Vermelho Amarelo tratado com lodo de esgoto, a quantidade de $\mathrm{Mn}$ encontrada, na maioria das partes das plantas, não diferiu da observada nas plantas cultivadas no solo sem aplicação do resíduo. Para o Latossolo Vermelho eutroférrico, a autora constatou que o teor disponível de $\mathrm{Mn}$ no solo foi reduzido com a aplicação do lodo de esgoto, entretanto a quantidade de $\mathrm{Mn}$, contida em várias partes das plantas, não apresentou diferença em relação ao tratamento sem aplicação do resíduo.

Oliveira (1995), ao correlacionar as quantidades de $\mathrm{Cu}$ absorvidas pelas plantas de milho com os teores removidos pelos extratores $\mathrm{HCl} 0,1 \mathrm{~mol} \mathrm{~L}^{-1}$ e DTPA, observou que ambos os extratores foram eficientes na avaliação da fitodisponibilidade de $\mathrm{Cu}$ em solos Neossolo Quartzarênico $\left(R^{2}=0,88\right.$ e $R^{2}=0,80$, respectivamente) e
Latossolo Vermelho eutroférrico $\left(R^{2}=0,88\right.$ e $R^{2}=0,88$, respectivamente) tratados com lodo de esgoto. Mulchi et al. (1991) estudaram o efeito residual da aplicação de lodo de esgoto sobre a disponibilidade de metais para a cultura do fumo, cultivada em solos franco siltoso e franco arenoso, que receberam, durante oito anos, doses de 0 , 56,112 e $224 \mathrm{Mg} \mathrm{ha}^{-1}$ (base seca). Os autores verificaram que, dos extratores avaliados (Mehlich-1, Mehlich-3 e DTPA) nenhum extrator se mostrou eficiente para o $\mathrm{Fe}$.

\section{CONCLUSÕES}

A aplicação de lodo de esgoto promoveu aumento nos teores disponíveis de $\mathrm{Fe}, \mathrm{Mn}, \mathrm{Zn}$ e $\mathrm{Cu}$ no solo, na produção de matéria seca e consequente aumento no acúmulo de $\mathrm{Fe}, \mathrm{Mn}, \mathrm{Zn}$ e $\mathrm{Cu}$ pelas plantas.

A complementação do resíduo com adubo potássico aumentou a produção de matéria seca da parte aérea das plantas e diminuiu o acúmulo de $\mathrm{Zn}$ pelas plantas.

A redução do $\mathrm{pH}$ e a quantidade de $\mathrm{Mn}$ nativo do solo influenciaram no aumento da disponibilidade de Mn.

Todas as três soluções extratoras foram eficientes para predição da fitodisponibilidade de $\mathrm{Zn}$ e $\mathrm{Cu}$. Para o $\mathrm{Mn}$, os mais eficientes foram o $\mathrm{HCl} 0,01 \mathrm{~mol} \mathrm{~L}^{-1}$ e o Mehlich-3, enquanto que, para o $\mathrm{Fe}$ os mais eficientes

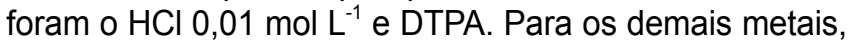
os extratores se mostraram pouco eficientes.

\section{REFERÊNCIAS BIBLIOGRÁFICAS}

ABREU, C.A.; ABREU, M.F.; RAIJ, B. van.; SANTOS, W.R. Comparação de métodos de análises para avaliar a disponibilidade de metais pesados em solos. Revista Brasileira de Ciência do Solo, v.19, p.463-468,1995.

ABREU, C.A. de; ABREU, M.F. de; SOARES, L.H.; ANDRADE, J.C. The effects of the DTPA extraction conditions on the determination of micronutrients in Brazilian soils. Communications in Soil Science and Plant Analysis, v.28, p.1-11, 1997.

ANJOS, A.R.M. Lixiviação de espécies químicas em latossolos sucessivamente tratados com biossólido e disponibilidade de metais pesados para plantas de milho. Piracicaba, 1999. 191p. Tese (Doutorado) - Escola Superior de Agricultura "Luiz de Queiroz", Universidade de São Paulo.

ANJOS, A.R.M.; MATTIAZZO, M.E. Metais pesados em plantas de milho cultivadas em Latossolos repetidamente tratados com biossólido. Scientia Agricola, v.57, p.769-776, 2000.

BERTONCINI, E.I. Mobilidade de metais pesados em solos tratados com lodo de esgoto. Piracicaba, 1997. 90p. Dissertação (Mestrado) - Escola Superior de Agricultura "Luiz de Queiroz", Universidade de São Paulo.

BORKERT, C.M. Manganês. In: SIMPÓSIO SOBRES MICRONUTRIENTES NA AGRICULTURA, 1., Jaboticabal, 1988. Anais. Piracicaba: POTAFOS; CNPq, 1991. p.173-190.

BUTING, A.H. Experiments on organic manures. Journal of Agricultural Science, v.60, p.121-140, 1963.

CAMARGO, O.A.; MONIZ, A.C.; JORGE, J.A.; VALADARES, J.M.A.S. Métodos de análise química, mineralógica e física de solos do Instituto Agronômico de Campinas. Campinas: IAC, 1986. 94p. (Boletim Técnico, 106).

CANTARELLA, H.; RAIJ, B. van.; QUAGGIO, J.A. Situação da análise de solo e planta no Brasil. In: REUNIÃO BRASILEIRA DE FERTILIDADE DO SOLO E NUTRIÇÃO DE PLANTAS, 21., Petrolina, 1994. Fertilizantes: insumo básico para agricultura e combate à fome; anais. Petrolina: EMBRAPA, CPATSA; SBCS; 1995. p.9-33.

CHANG, A.C.; HINESLY, T.D.; DONER, H.E.; DOWDY, R.H.; RYAN, J.A. Effects of long term sludge application on accumulation of trace elements by crops. In: PAGE, A.L.; LOGAN, T.J.; RYAN, J.A. Land application of sludge-food chain implications. Chelsea: Lewis Publisher, 1987. cap.4, p.53-66. 
EGREJA FILHO, F.B. Avaliação da ocorrência e distribuição química de metais pesados na compostagem do lixo domiciliar urbano. Viçosa, 1993. 176p. (Mestrado) - Universidade Federal de Viçosa.

ELLIOT, H.A.; LIBERATTI, M.R.; HUANG, C.P. Effect of iron oxide removal on heavy metal sorption by acid subsoils. Water, Air and Soil Pollution, v.37, p.379-389, 1986.

EMPRESA BRASILEIRA DE PESQUISA AGROPECUÁRIA. Manual de métodos de análise de solo 2.ed. Rio de Janeiro: EMBRAPA, CNPS, 1997. 212p.

HOODA, P.S.; ALLOWAY, B.J. The plant availability and DTPA extractability of trace metals in sludge-amended soils. The Science of the Total Environment, v.149, p.39-51, 1993.

HOODA, P.S.; McNULTY, D.; ALLOWAY, B.J.; AITKEN, M.N. Plant availability of heavy metals in soils previously amended with heavy applications of sewage sludge. Journal of Science of Food and Agriculture, v.73, p.446454, 1997.

KABATA-PENDIAS, A.; PENDIAS, H. Trace elements in soil and plants. Boca Raton: CRC Press, 1984. 315p.

KIEHL, E.J. Fertilizantes orgânicos. Piracicaba: Ceres, 1985. 492p.

LESCHBER, R.; DAVIES, R.D.; L'HERMITE, P. Chemical methods for assessing bioavailability metals in sludge and soils. London: Elsevier, 1985. 96p.

LINDSAY, W.L.; NORWEL, W.A. Development of a DTPA soil test for zinc, iron, manganese and copper. Soil Science Society of America Journal, v.42, p.421-428, 1978.

MALAVOLTA, E.; VITTI, G.C.; OLIVEIRA, S.A. Avaliação do estado nutricional das plantas: princípios e aplicações. 2.ed. Piracicaba: Potafos, 1997. 319p.

MEHLICH, A. Mehlich-3 soil test extraction: A modification of Mehlich-2. Communications in Soil Science and Plant Analysis, v.15, p.14091416, 1984.

MULCHI, C.L.; ADAMU, C.A.; BELL, P.F.; CHANEY, R.L. Residual heavy metal concentrations in sludge-amended coastal plain soils: I. Comparason of extractants. Communications in Soil Science and Plant Analysis, v.22, p.919-941, 1991.

OLIVEIRA, F.C. Metais pesados e formas nitrogenadas em solos tratados com lodo de esgoto. Piracicaba, 1995. 90p. Dissertação (Mestrado) Escola Superior de Agricultura "Luiz de Queiroz", Universidade de São Paulo.
OLIVEIRA, F.C. Disposição de lodo de esgoto e composto de lixo num Latossolo Vermelho-amarelo cultivado com cana-de-açúcar. Piracicaba, 2000. 247p. Tese (Doutorado) - Escola Superior de agricultura "Luiz de Queiroz", Universidade de São Paulo.

OLIVEIRA, F.C.; MARQUES, M.O.; BELLINGIERI, P.A.; PERECIN, D. Lodo de esgoto como fonte de macronutrientes para a cultura do sorgo granífero. Scientia Agricola, v.52, p.360-367, 1995.

RAIJ, B. van; QUAGGIO, J.A. Métodos de análise de solo para fins de fertilidade. Campinas: IAC, 1983. 40p. (Boletim Técnico, 81).

RAIJ, B van; SILVA, N.M; BATAGLIA, O.C.; QUAGGIO, J.A; HIROCE, R CATARELLA, H.; BELLINAZZI JR., R.; DECHEN, A.R.; TRANI, P.E. Recomendação de adubação e calagem para o Estado de São Paulo Campinas: IAC, 1996. 285p. (Boletim técnico, 100)

ROCA, J.; POMARES, F. Prediction of available heavy metals by six chemical extractants a sewage sludge-amended soil. Communications in Soil Science and Plant Analysis, v.22, p.2129-2136, 1991.

SARRUGE, J.R.; HAAG, H.P. Análises químicas em plantas. Piracicaba: ESALQ, 1974. 56p.

SAUERBECK, D.R.; STYPEREK, P. Evaluation of methods for assessing the $\mathrm{Cd}$ and $\mathrm{Zn}$ availability from different soils and sources. In: LESCHBER, R.; DAVIES, R. D.; L'HERMITE, P. (Ed.) Chemical methods for assessind biovailability metal in sludge and soils. London: Elsevier, 1985. p. 49 66

SHUMAN, L.M. Effect of ionic strength and anions on zinc adsorption by two soils. Soil Science Society of America Journal, v.50, p.1438-1442, 1986.

SOLTANPOUR, P.N.; JONES JR., J.; WORKMAN, S.M. Optical emission spectrometry. In: PAGE, A.L.; MILLER, R.H.; KEENEY, D.R. (Ed.) Methods of soil analysis. Madison: ASA; SSSA, 1982. P.29-65.

TRAN, T.S.; GIROUX, M.; GUILBEAUT, J.; AUDES, P. Evaluation of MehlichIII extractant to estimate the available P in Quebec Soils. Communications in Soil Science and Plant Analysis, v.21, p.1-28, 1990.

TAYLOR, R.W.; IBEABUCHI, I.O.; SISTANI, K.R.; SHUFORD, J.W. Heavy metal concentration in forage and extractabilility from some acid mine spoils. Water, Air and Soil Pollution, v.68, p.363-372, 1993.

TISDALE, S.L.; NELSON,W.L.; BEATON, J.D. Soil fertility and fertilizers. 4.ed. New York: Macmillan,1985. 754p.

Recebido em 04.04.01 